\title{
Spontaneous Development of Left Ventricular Aneurysm in a Patient With Untreated Cardiac Sarcoidosis
}

\author{
Tatsuo Haraki, MD; Kousei Ueda, MD; Hiromoto Shintani, MD; \\ Tatsumi Hayashi, MD; Junichi Taki, MD*; Hiroshi Mabuchi, MD**
}

\begin{abstract}
A young adult patient with untreated sarcoidosis spontaneously developed a left ventricular (LV) aneurysm in the anterolateral free wall. Single-photon emission computed tomography (SPECT) using Gallium-67 clearly demonstrated widespread abnormal uptake, including the LV aneurysm. Thallium-201 SPECT revealed a perfusion defect in the anterolateral wall, and abnormal uptake of technetium-99m pyrophosphate was seen, especially in the borders of the defect lesion. (Circ J 2002; 66: 519-521)
\end{abstract}

Key Words: Cardiac sarcoidosis; Gallium-67 single-photon emission computed tomography (SPECT); Left ventricular aneurysm

$\mathbf{S}$ arcoidosis is a systemic granulomatous disorder of unknown origin with a poor prognosis in patients with cardiac involvement because of the associated sudden death, fatal cardiac tachyarrhythmia, conduction block, and heart failure ${ }^{-3}$ Therefore, early diagnosis and corticosteroid therapy are important in patients with cardiac sarcoidosis, 4 but clearly establishing cardiac involvement can be difficult in the early stages of the disease. The cardiac lesions are usually focal and scattered, mostly in the interventricular septum (IVS) and left ventricular wall!-3 Extension of the ventricular lesions can lead to aneurysm formation and these have been reported as 'healed granuloma' after corticosteroid therapy! We report here a case of cardiac sarcoidosis in which a left ventricular (LV) aneurysm developed spontaneously, not after corticosteroid therapy.

\section{Case Report}

A 27-year-old man was referred for evaluation of an abnormal chest shadow detected in a routine $\mathrm{X}$-ray examination. There were diffuse infiltrative shadows in both lung fields, no hilar lymphadenopathy and a normal cardiac shadow. The serum concentration of angiotensin-converting enzyme (ACE) was increased (32.0 U/L; normal range, $7.0-25.0 \mathrm{U} / \mathrm{L})$, as was the concentration of lysozyme (16.9 $\mu \mathrm{g} / \mathrm{ml}$; normal range, $5.0-10.9 \mu \mathrm{g} / \mathrm{ml}$ ). Other laboratory findings were normal. An electrocardiogram (ECG) showed right axis deviation, poor progression of the $\mathrm{R}$ wave in leads $\mathrm{I}$ and $\mathrm{a} \mathrm{V}_{\mathrm{L}}$, and incomplete right-bundle branch block (Fig 1A). Echocardiography revealed mild hypokinesis in the LV anterior wall with normal thickness of the IVS (7 $\mathrm{mm})$ and posterior wall $(8 \mathrm{~mm})$. The LV ejection fraction

(Received May 14, 2001; revised manuscript received July 2, 2001; accepted July 17, 2001)

Department of Internal Medicine, Komatsu Municipal Hospital, Komatsu, and *Department of Nuclear Medicine and **The Second Department of Internal Medicine, Kanazawa University, Kanazawa, Japan

Mailing address: Tatsuo Haraki, MD, Department of Internal Medicine, Komatsu Municipal Hospital, 60 Ho, Mukaimoto-ori, Komatsu 923-8560, Japan. E-mail: tatsuo.h@p2242.nsk.ne.jp
(LVEF) was $60 \%$. The ratio of CD4/CD8 cells in bronchoalveolar lavage fluid was increased to 11.6. Noncaseating sarcoid granuloma was confirmed by transbronchial lung biopsy (TBLB). Although abnormal uptake of gallium$67\left({ }^{67} \mathrm{Ga}\right)$ was seen in both lung fields and the right paratra-

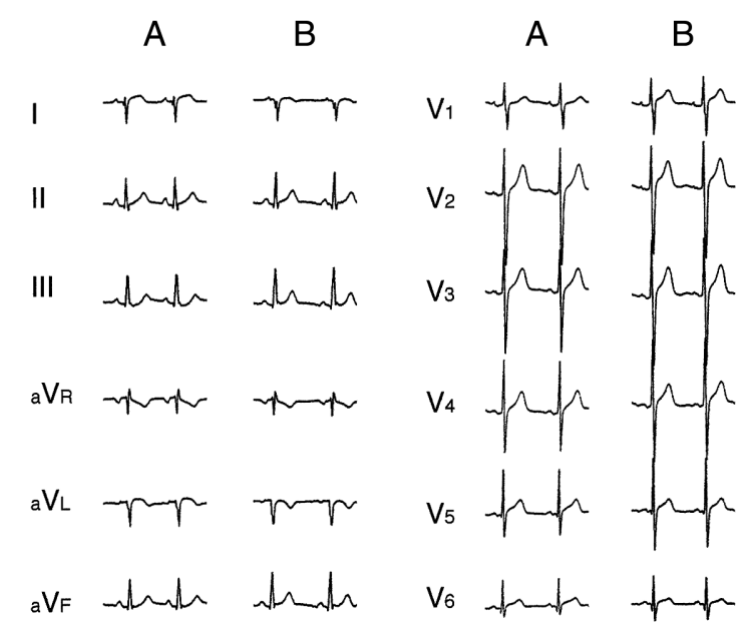

Fig 1. Electrocardiogram at the age of 27 years (A) and after 18 months of follow up (B). An abnormal $R$ wave in the $I$ and $a V_{L}$ leads gradually developed.

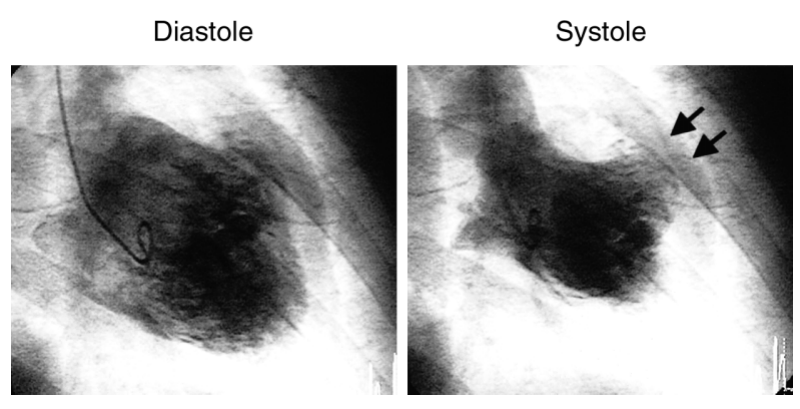

Fig 2. Left ventriculography show an aneurysm (triangle) in the anterolateral wall. 


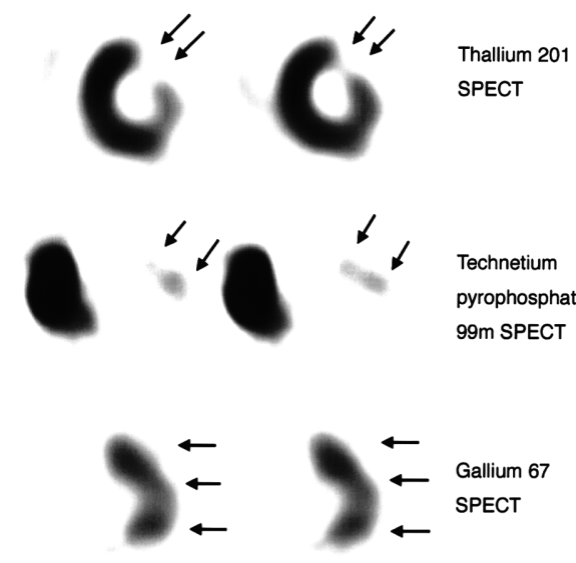

Fig 3. Short-axis thallium-201 (201Tl) images, technetium-99m pyrophosphate (99mTc-PYP), and Gallium-67 ( $\left.{ }^{67} \mathrm{Ga}\right)$ single-photon emission computed tomography (SPECT) before corticosteroid therapy. ${ }^{201} \mathrm{Tl}$ SPECT shows a perfusion defect in the left ventricular anterolateral wall, and abnormal uptake of $99 \mathrm{mTc}-\mathrm{PYP}$ can be seen, especially in the borders of the defect lesion. ${ }^{67} \mathrm{Ga}$ SPECT demonstrated widespread abnormal uptake in the anterolateral and inferior walls of the left ventricle.

cheal lymph node, it was not seen in the cardiac system and so because cardiac involvement was not suspected at that time, the patient was followed without steroid therapy.

About 18 months later, the concentrations of both ACE and lysozyme were still increasing (26.4 U/L and 15.4 $\mu \mathrm{g} / \mathrm{ml}$, respectively) and the diffuse infiltrative shadows in the lung fields were still present. None of the other body organs (such as skin, muscle, eyes and nervous system) appeared to be involved. ECG showed an abnormal Q wave with a decrease in the $\mathrm{R}$ wave in the $\mathrm{I}$ and $\mathrm{aVL}$ leads (Fig 1B), but Holter ECG monitoring did not detect ventricular tachyarrhythmia or conduction block. Echocardiography revealed severe hypokinesis and dyskinesis extending to the LV anterolateral wall, which had become thin. Moreover, there was a new, small hypokinetic lesion in the posterior wall. The LVEF had decreased to $45 \%$, so we performed cardiac catheterization because we now suspected cardiac involvement. The coronary angiogram showed normal coronary arteries, but left ventriculography (LVG) revealed an aneurysm in the anterolateral free wall (Fig 2). Noncaseating sarcoid granuloma was not found in an endocardial biopsy from the right ventricle. Planar ${ }^{67} \mathrm{Ga}$ scintigraphy indicated that the abnormal uptake in both lung fields had reduced compared with that seen previously, but the abnormal uptake of ${ }^{67} \mathrm{Ga}$ by the cardiac system was still inconclusive. However, ${ }^{67} \mathrm{Ga}$ single-photon emission computed tomography (SPECT) clearly demonstrated widespread abnormal uptake in the anterolateral and inferior walls of the LV (Fig 3). Thallium-201 (201Tl) SPECT revealed a perfusion defect in the anterolateral wall, and abnormal uptake of technetium-99m pyrophosphate ( $99 \mathrm{mTc}$ PYP) was seen, especially in the borders of the defect lesion (Fig 3). Therefore, we instituted corticosteroid therapy at an initial daily dose of $30 \mathrm{mg}$ of prednisolone. Two months later, echocardiography showed that the severe hypokinesis and thinning of the LV anterolateral wall were still present, but the wall motion of the posterior wall had improved.

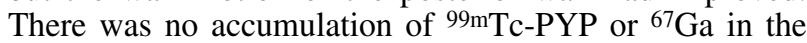
cardiac lesion, but the perfusion defect in the anterolateral wall was still present on ${ }^{201} \mathrm{Tl}$ SPECT, which suggested non-reversible aneurysmal change.

\section{Discussion}

The clinical diagnosis of cardiac involvement in sarcoidosis is difficult in the early stage of the disease. Autopsy studies have revealed cardiac involvement in approximately $25-78 \%$ of patients, ${ }^{2-4}$ and it is more frequently observed in Japanese patients.-4 Endocardial biopsy often fails to diagnose it, and although Holter ECG monitoring can predict it, the sensitivity and specificity are relatively low6 Therefore, myocardial perfusion imaging has been used. ${ }^{201} \mathrm{Tl}$ SPECT can detect myocardial defects and can be used to evaluate the size of the lesion? An abnormal ${ }^{67} \mathrm{Ga}$ uptake can localize the cardiac involvement and indicate the likely response to corticosteroid therapy ${ }^{8-11}$ The present case demonstrates the difficulty in establishing cardiac involvement in the early stage of sarcoidosis. There were not any symptoms of heart failure or ventricular arrhythmia and cardiac involvement was suspected on the basis of trivial ECG changes and LV functional abnormality on echocardiography. The abnormal cardiac uptake seen on planar ${ }^{67} \mathrm{Ga}$ scintigraphy was equivocal, and ${ }^{67} \mathrm{Ga}$ SPECT could only just confirm cardiac involvement.

It is rare for a LV aneurysm to develop without concomitant coronary artery disease ${ }^{12}$ and if normal cardiac function is present, the prognosis for an aneurysm caused by myocarditis is not poor ${ }^{3}$ In contrast, the prognosis for aneurysm caused by cardiac sarcoidosis is uncertain because of its rarity!4-18 Fibrosis and degeneration of the ventricle wall may result in protrusion and then formation of an aneurysm! ${ }^{-3}$ Autopsy studies indicate that the frequency of aneurysm formation is approximately $8 \%$ of patients with cardiac involvement of sarcoidosis! The wall of the aneurysm does not always contain sarcoid granulomas and fibrous tissue predominates, ${ }^{1-3}$ which suggests long-standing cardiac involvement. Steroid therapy can heal a sarcoid granuloma and accelerate fibrous change, but such healing would be expected to occur without treatment.

There was no histological evidence of sarcoid granuloma in the endomyocardial biopsy of the right ventricle, and we did not the left ventricle biopsy. However, based on the perform results of TBLB and the clinical course, we believe that the LV aneurysm was a clinical manifestation of cardiac sarcoidosis. Therefore, it is a dilemma when administration of corticosteroids should be started. There was abnormal uptake of $99 \mathrm{mTc}-\mathrm{PYP}$, especially in the borders of the defect lesion observed on ${ }^{201} \mathrm{Tl}$ SPECT, before steroid therapy was begun in the present case. Accumulation of $99 \mathrm{mTc}-\mathrm{PYP}$ reflects the absence of calcium and the presence of denatured proteins in myocardial cells, indicative of an active aneurysm. In contrast, ${ }^{67} \mathrm{Ga}$ SPECT indicated that the abnormal uptake was widespread, including the LV aneurysm. Thus, the LV aneurysm may have contained an active sarcoid granuloma with scar tissue. After steroid therapy, there was no uptake of $99 \mathrm{mTc}-\mathrm{PYP}$ or ${ }^{67} \mathrm{Ga}$ in the cardiac lesion, and although the perfusion defect detected on ${ }^{201} \mathrm{Tl}$ was still present, it had not extended. Corticosteroid therapy prevented expansion of the LV aneurysm, but further careful follow-up is needed for this patient.

\section{References}

1. Roberts WC, McAllister HA, Ferrans VJ. Sarcoidosis of the heart: A clinicopathologic study of 35 necropsy patients (group 1) and review of 78 previously described necropsy patients (group 11). Am J Med 
1977; 63: 86-108.

2. Silverman KJ, Hutchins GM, Bulkley BH. Cardiac sarcoid: A clinicopathologic study of 84 unselected patients with systemic sarcoidosis. Circulation 1978; 58: 1204-1211.

3. Matsui Y, Iwai K, Tachibana T, Fruie T, Shigematsu N, Izumi T, et al. Clinicopathological study of fatal myocardial sarcoidosis. Ann NY Acad Sci 1976; 278: 455-469.

4. Sekiguchi M, Yazaki Y, Isobe M, Hiroe M. Cardiac sarcoidosis: Diagnostic, prognostic, and therapeutic considerations. Cardiovasc Drugs Ther 1996; 10: 495-510.

5. Uemura A, Morimoto S, Hiramitsu S, Kato Y, Ito T, Hishida H. Histologic diagnostic rate of cardiac sarcoidosis: Evaluation of endomyocardial biopsies. Am Heart J 1999; 138: 299-302.

6. Suzuki T, Kanda T, Kubota S, Imai S, Murata K. Holter monitoring as a non-invasive indicator of cardiac involvement in sarcoidosis. Chest 1994; 106: 1021-1024.

7. Bulkley BH, Rouleau JR, Whitaker JQ, Strauss W, Pitt B. The use of 201 thallium for myocardial perfusion imaging in sarcoid heart disease. Chest 1977; 72: 27-32.

8. Forman MB, Sandler MP, Sacks GA, Kronenberg MW, Powers TA Radionuclide imaging in myocardial sarcoidosis: Demonstration of myocardial uptake of technetium pyrophosphate $99 \mathrm{~m}$ and gallium. Chest 1983; 83: $578-580$.

9. Taki J, Nakajima K, Bunko H, Ohguchi M, Tonami N, Hisada K. Cardiac sarcoidosis demonstrated by Tl-201 and Ga-67 SPECT imaging. Clin Nucl Med 1990; 15: 636-639.

10. Tawarahara K, Kurata C, Okayama K, Kobayashi A, Yamazaki N. Thallium-201 and gallium 67 single photon emission computed tomographic imaging in cardiac sarcoidosis. Am Heart J 1992; 124: $1383-1384$

11. Yazaki Y, Isobe M, Hayasaka M, Tanaka M, Fujii T, Sekiguchi M. Cardiac sarcoidosis mimicking hypertrophic cardiomyopathy: Clinical utility of radionuclide imaging for differential diagnosis. Jpn Circ J 1998; 62: 465-468.

12. Toda G, Illiev II, Kawahara F, Hayano M, Katsusuke Y. Left ventricular aneurysm without coronary artery disease, incidence and clinical features: Clinical analysis of 11 cases. Intern Med 2000; 39: 531 536.

13. Andrea F, Cristina C, Maurizio P. Prognostic significance of left ventricular aneurysms with normal global function caused by myocarditis. Chest 2000; 118: 1696-1702.

14. Lull RJ, Dunn BE, Gregoratos G, Cox WA, Fisher GW. Ventricular aneurysm due to cardiac sarcoidosis with surgical cure of refractory ventricular tachycardia. Am J Cardiol 1972; 30: 282-287.

15. Chun SK, Andy JJ, Jilly P. Ventricular aneurysm in sarcoidosis. Chest 1975; 68: 392-393.

16. Ahmed SS, Rozefort R, Taclob LT, Blancato RW. Development of ventricular aneurysm in cardiac sarcoidosis. Angiology 1977; 28: $323-329$.

17. Jain A, Starek PJ, Delany DL. Ventricular tachycardia and ventricular aneurysm due to unrecognized sarcoidosis. Clin Cardiol 1990; 13: $738-740$.

18. Kosuge H, Noda M, Kakuta T, Kishi Y, Isobe M, Numano F. Left ventricular apical aneurysm in cardiac sarcoidosis. Jpn Heart J 2001; 42: $265-269$. 